\title{
Pre-stressed Curved Actuators: Characterization and Modeling of their Piezoelectric Behavior
}

\author{
Karla Mossi ${ }^{1}$, Zoubeida Ounaies ${ }^{1}$, Ralph Smith ${ }^{2}$ and Brian Ball ${ }^{2}$ \\ ${ }^{1}$ Virginia Commonwealth University, Richmond, VA 23284-3015 \\ ${ }^{2}$ CRS C, North Carolina State University, Raleigh, NC 27695
}

\begin{abstract}
Pre-stressed curved actuators consist of a piezoelectric ceramic (lead zirconate titanate or PZT) sandwiched between various substrates and other top layers. The substrates used in this study are stainless steel, and fiberglass. The top layers are made up of aluminum and carbon. Due to their enhanced strain capabilities, these pre-stressed piezoelectric devices are of interest in a variety of aerospace applications. Their performance as a function of electric field, temperature and frequency is needed in order to optimize their operation. During the processing steps, a mismatch between the properties of the various layers leads to pre-stressing of the PZT layer. These internal stresses, combined with restricted lateral motion, are shown to enhance the axial displacement. The goal is to gain an understanding of the resulting piezoelectric behavior over a range of voltages, and frequencies, for unloaded conditions. To quantify the relationship between the state of stress in the ceramic and the overall performance, a physics-based model is developed incorporating both thermo elastic relations and ferroelectric domain theory. The model predicts displacements based on the geometry and physical characteristics of the actuator components. The accuracy of the model and associated numerical method is demonstrated through comparison with experimental data for various input voltages, and boundary conditions.
\end{abstract}

Keywords: piezoelectric actuators, Lipca, Thunder, displacement performance

\section{INTRODUCTION}

Pre-stressed curved metal actuators are composites made of a ceramic or active layer, and one or more inactive layers, usually metallic. The active layer is composed of a piezoelectric ceramic (PZT5A), and the metallic layer is commonly aluminum, stainless steel, or brass [i]. The actuator size has been determined according to the desired end use, either for research or for the development of an application [ii]. One of the design parameters used is the thickness ratio of active to inactive layer for several elements with different cross sectional areas, and different metals [iii, iv]. A description of the manufacturing process can be found elsewhere [v]. The devices are pre-stressed due to the different coefficients of thermal expansion of the layers since the composite is processed at high temperatures, $300{ }^{\circ} \mathrm{C}$. As demonstrated by numerous studies [vi, vii, viii], this process has a confirmed enhanced displacement as compared to a PZT Unimorph. A new type of actuator has been designed based on the same principle described above, where the metallic layers has been substituted with fiberglass and unidirectional carbon [ix, $\mathrm{x}$ ].

This study concentrates on studying the differences in displacement performance between these new types of actuators named Lipca ${ }^{1}$, and the metallic ones, called Thunder ${ }^{2}$. Displacement performance under specific boundary conditions at different fields and frequencies are tested.

\footnotetext{
${ }^{1}$ Manufactured by Konkuk University, South Korea

${ }^{2}$ Manufactured by Face International, Norfolk, VA
} 


\section{EXPERIMENTAL SETUP}

Experiments are conducted to consistently quantify the free-displacement of different types of pre-stressed piezoelectric actuators. The groups tested consist of the actuators, LIPCA-C2 and Thunder. Lipca actuators are composed of a glass/epoxy top layer of 3.937 in x 0.945 in x 0.035 in, a layer of unidirectional carbon epoxy layer of 2.795 in x 0.866 in x 0.004 in, another glass/epoxy layer, a PZT layer of 2.85 inx 0.906 in $x 0.0098$ in, and another glass/epoxy layer, as shown in Figure 1. Thunder devices consist of a top aluminum layer 2.795 in x 0.945 in x 0.001 in, an SI adhesive layer, a PZT layer of 2.85 in x 0.945 in $x$ 0.0091 in type 3195HD (CTS wireless), another adhesive layer, and a stainless steel bottom layer of 3.819 in $x 0.945$ in x 0.0074 in, as shown in Figure 2. There are 10 samples per case.

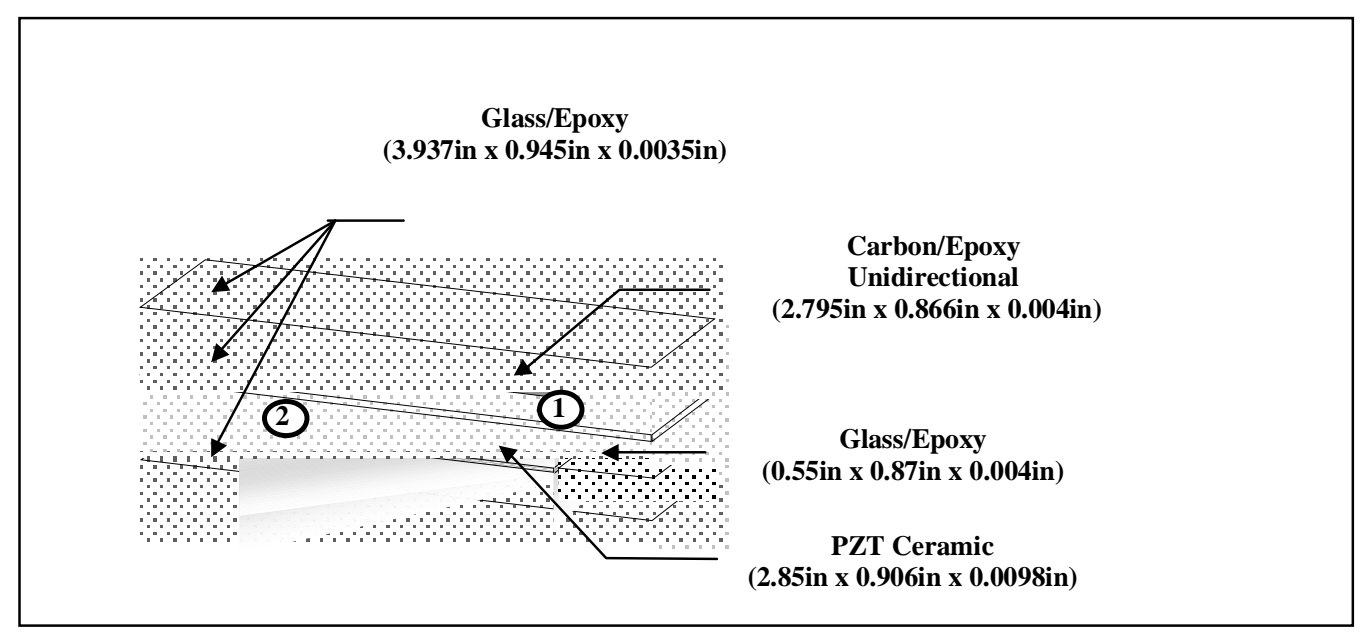

Figure 1. Layer composition of a Lipca-C2 Device

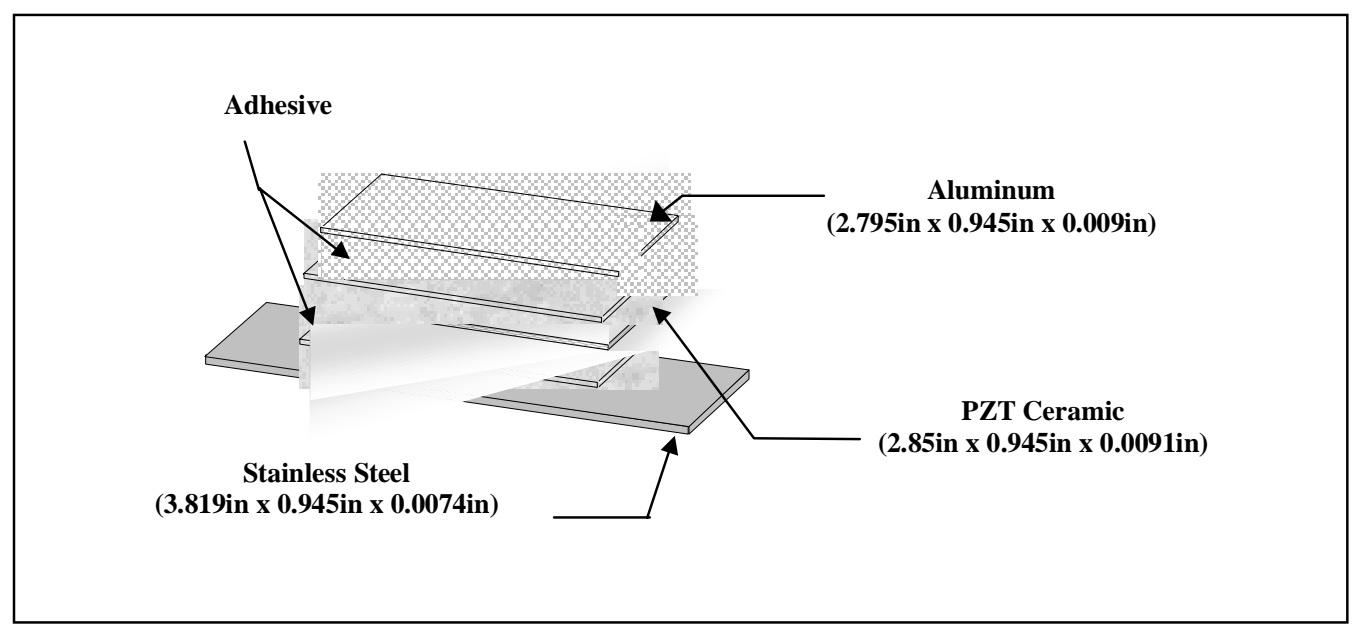

Figure 2. Layer composition of a Thunder-C2 Device

The actuators are measured using a Multitoyo micrometer, a balance, an HP4194 impedance analyzer, a NAIS LM10 non-contact laser, an Intel digital optical microscope, a LeCroy 9350L oscilloscope, an HP 33120A signal generator, a TREK PZD700 voltage amplifier, and a data acquisition system. Furthermore, a specially designed fixture weighing approximately $10 \mathrm{lbs}$ was built that consisted of a block of steel, a fixed support in one end, and a linear bearing by American Linear Manufacturers Inc. model LPA12-1-05 
at the other end, providing a pin-free simply supported holder (Figure 3). This setup provides consistent boundary conditions, namely a pin and a roller, and enough support for the actuators to be operated at resonant frequencies.

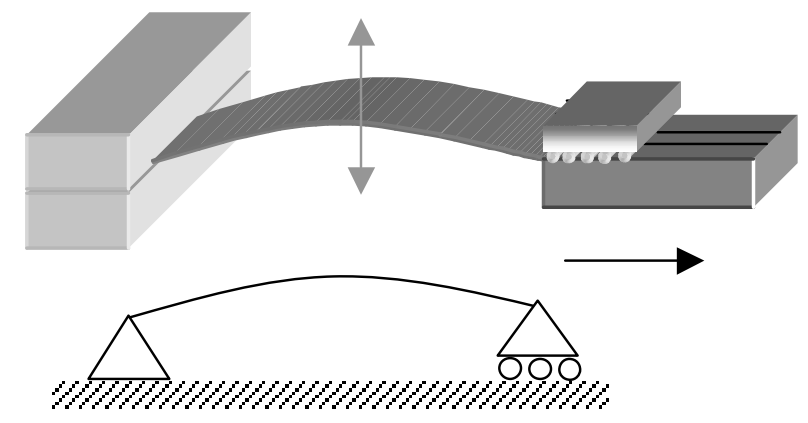

Figure 3. Schematic of holding fixture used for testing

\section{RESULTS}

The first set of measurements performed included weighing each actuator, measuring total thickness, and measuring dome height. These measurements are then averaged and a summary of these results is shown in Table 1. It can be clearly seen that Lipca-C2 weighs 1.4 times less than a Thunder device of the approximate same cross sectional area, even though the total thickness is different. In short a Lipca device is 31\% lighter than Thunder. The total thickness of Lipca is 35\% higher than that of a Thunder device. The active layer, the PZT, has approximately the same dimensions for both sets of devices. The dome-height of a device, defined as the tallest point of the curved device measured with a non-contact laser, shows that Lipca is $65 \%$ flatter than a Thunder device.

This last measurement is usually a good indication of the amount of pre-stress in the Thunder device and has been used as one gauge of the performance of the final device [3].

Table 1 Static Measurement Comparison

\begin{tabular}{|l|c|c|c|}
\cline { 2 - 4 } \multicolumn{1}{c|}{} & Weight (oz) & $\begin{array}{c}\text { Total Thickness } \\
\text { (mils) }\end{array}$ & $\begin{array}{c}\text { Dome Height } \\
\text { (mils) }\end{array}$ \\
\hline & $\overline{\boldsymbol{m}} \pm \boldsymbol{\sigma}_{\boldsymbol{m}}$ & $\overline{\boldsymbol{t}} \pm \boldsymbol{\sigma}_{\boldsymbol{t}}$ & $\overline{\boldsymbol{h}} \pm \boldsymbol{\sigma}_{\boldsymbol{h}}$ \\
\hline Lipca-C2 & $0.192 \pm 0.0019$ & $29.17 \pm 0.346$ & $83.23 \pm 9.96$ \\
\hline Thunder & $0.279 \pm 0.0046$ & $18.90 \pm 1.362$ & $238.07 \pm 6.38$ \\
\hline
\end{tabular}

$\sigma:$ standard deviation. Subscripts m: mass, $t$ : thickness, $h$ : dome height.

The next set of measurements, capacitance, dissipation factor, and impedance variations with frequency, are made using the impedance analyzer at $1 \mathrm{~V}_{\mathrm{rms}}(2.828 \mathrm{Vpp})$ signal level. From these results, dielectric loss and capacitance can be calculated and plotted. For the Lipca-C2 pieces, measurements were made using two sets of leads provided; one set has the top lead making contact with the ceramic through one layer of glass epoxy, while in the other set the top layer is in direct contact with the ceramic layer. The remaining contact for both sets of leads is through the glass epoxy layer at the bottom of the configuration. These leads correspond to no. 1 and no. 2 respectively and are marked on Figure 2. All the Lipca samples were 
measured using the two set of leads. Because of the scatter on the results obtained, no averages were calculated. Differences in capacitance among samples are seen between 100-600 Hz ranges, but not at the higher frequencies. Since the frequencies of interest for the displacement performance measurements are all below $1 \mathrm{kHz}$, the no. 2 set of leads was utilized to provide higher readings of capacitance below $1 \mathrm{kHz}$. For Thunder samples, the results are more uniform, however averages eliminate any frequency peaks present.

Both capacitance and dissipation were measured for both groups for the same range of frequencies. From these results, dielectric constant and dielectric loss factor are calculated based on the dimensions. A typical result for dielectric constant variations with frequency for a Thunder and Lipca sample are shown in Figures 4 and 5 respectively. Note that the peaks obtained for both type of devices are located at the same frequencies. However, for a typical Thunder piece the magnitudes of these peaks are much smaller. This may be due to the presence of the adhesive utilized for manufacturing a Thunder device, since the measurement of capacitance is done through the adhesive layer. This dielectric mismatch between the adhesive, LaRC-SI and the PZT is greater than 300. This indicates that for the PZT in Thunder to see the same applied field than Lipca, a higher voltage needs to be applied. The plots of the dielectric loss reinforce these results (see Figures 6 and 7 for Lipca and Thunder, respectively).

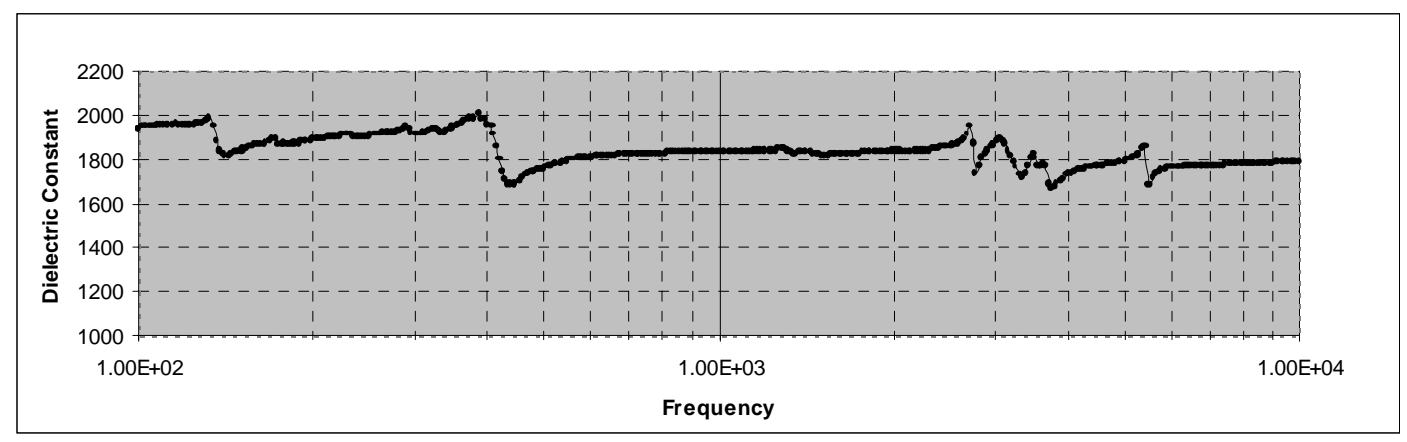

Figure 4. Dielectric Constant variations with frequency for a typical Lipca-C2 device

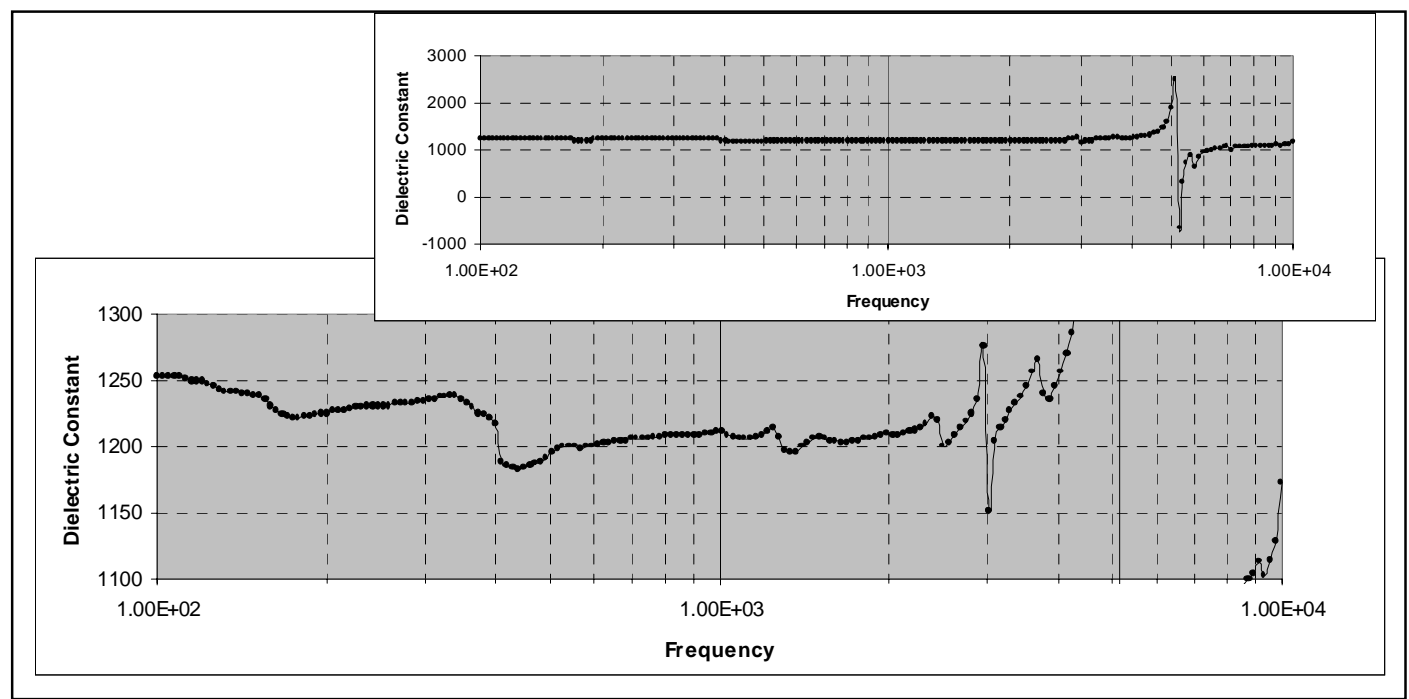

Figure 5. Dielectric Constant variations with frequency for a typical Thunder device 


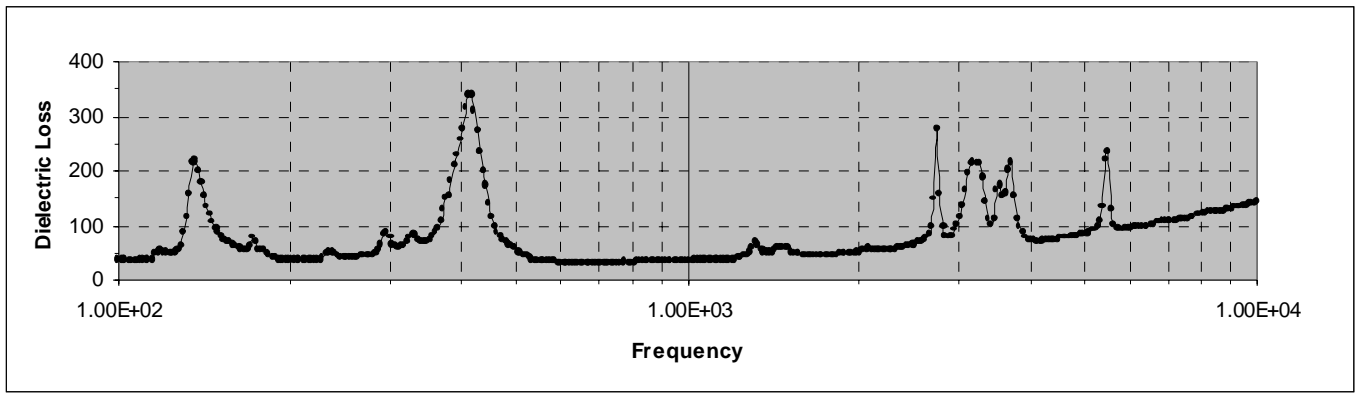

Figure 6. Dielectric loss variations with frequency for a typical Lipca Sample

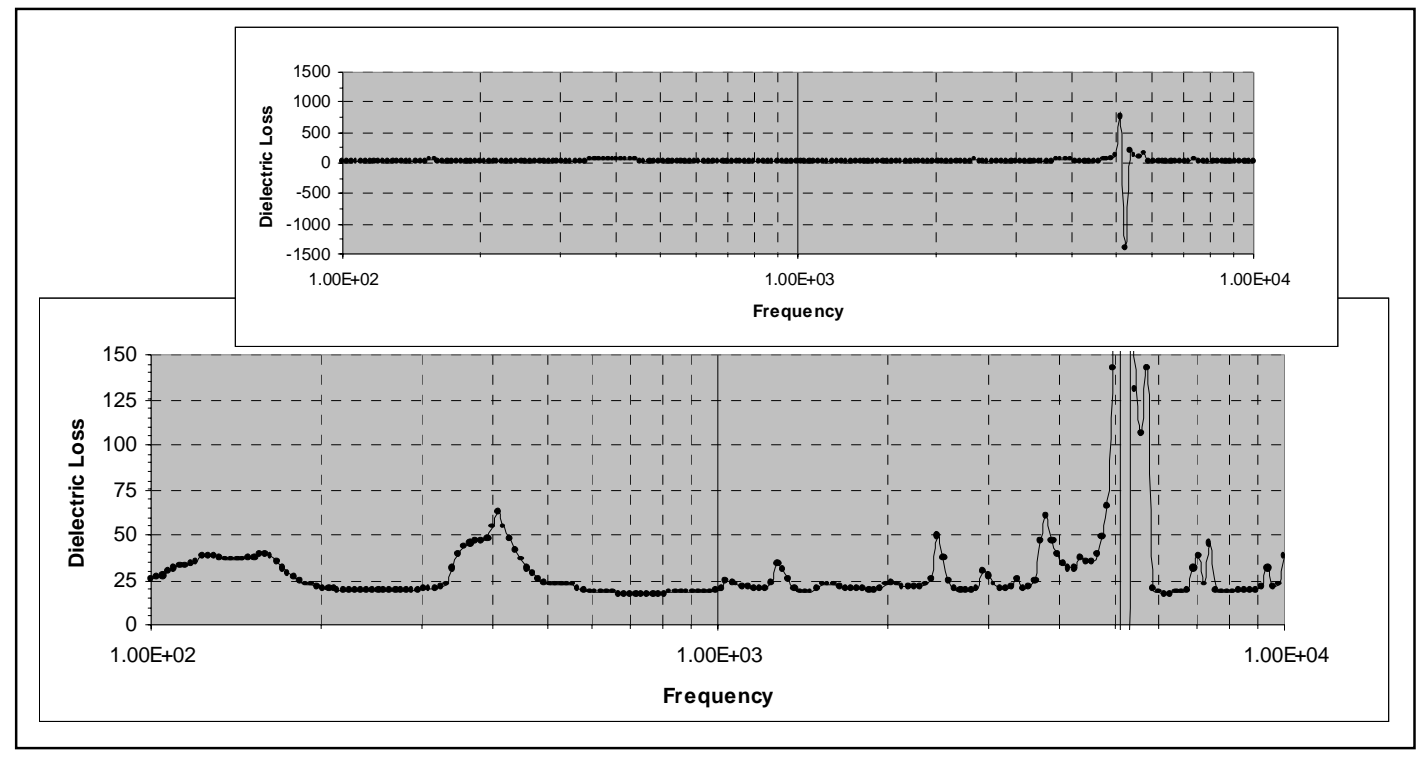

Figure 7. Dielectric loss variations with frequency for a typical Thunder Sample

Impedance and phase angle were also measured as a function of frequency. From these plots, resonant frequency for both samples can be identified noting that these values are measured at 1 Vrms. For both devices, the resonant points are all below $400 \mathrm{~Hz}$. For this reason the results are plotted for the range of 100 to $1000 \mathrm{~Hz}$. The magnitudes of the peaks again for Thunder are smaller than for Lipca; Lipca has several peaks at $135,155,256,265,285$, sand $320 \mathrm{~Hz}$; for Thunder, there are only two peaks at 140 and $310 \mathrm{~Hz}$. As will be shown later, these values correspond to resonance as detected by the displacement measurements. Results are shown in Figures 8 and 9 respectively. 


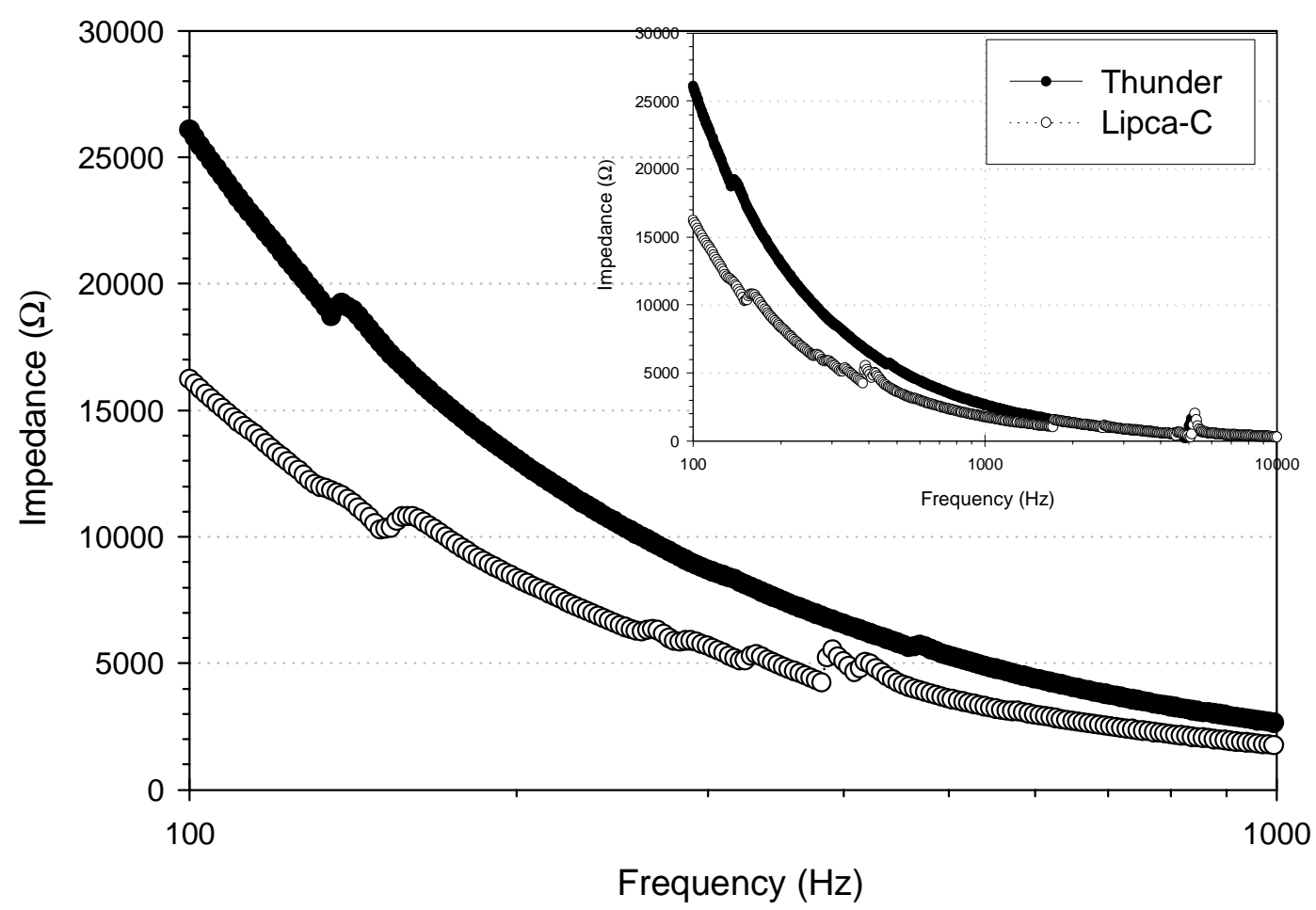

Figure 8. Impedance variations with frequency for a typical thunder and Lipca sample

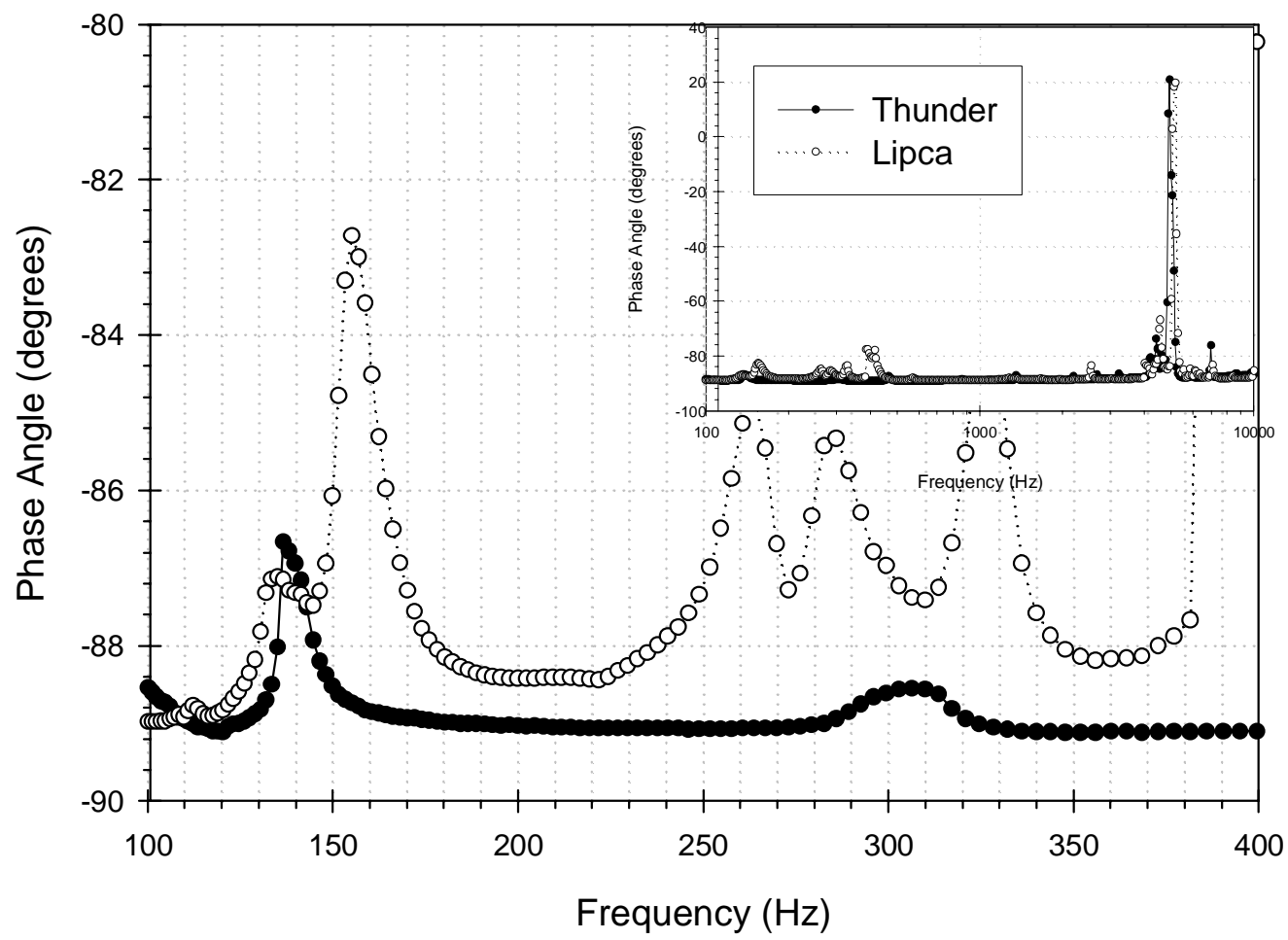

Figure 9. Phase angle variations with frequency for a typical thunder and Lipca sample 
Out-of-plane displacement measurements were performed using a non-contacting Laser technique. Sinusoidal voltages were applied and peak voltages varied from $100 \mathrm{~V}, 150 \mathrm{~V}, 200 \mathrm{~V}$, and $250 \mathrm{~V}$ at frequencies ranging from $1 \mathrm{~Hz}$ to $300 \mathrm{~Hz}$. A typical voltage displacement loop at $1 \mathrm{~Hz}$ for a Lipca sample is shown in Figure 10. As can be seen, the loop is hysteretic in nature owing to the piezoelectric nature of the active layer. In contrast, a Thunder piece is shown in Figure 11 also at $1 \mathrm{~Hz}$ and selected voltages. It is clear from the results that the Lipca devices produced higher displacement. Also, a typical Lipca loop at 1 $\mathrm{Hz}$ is more symmetrical and less noisy than a Thunder sample. This may be due to the higher magnitude displacement that the Lipca pieces exhibited (and is therefore related to the resolution of the Laser device used). It is important to note that the Lipca pieces are experiencing a higher field since the electric leads

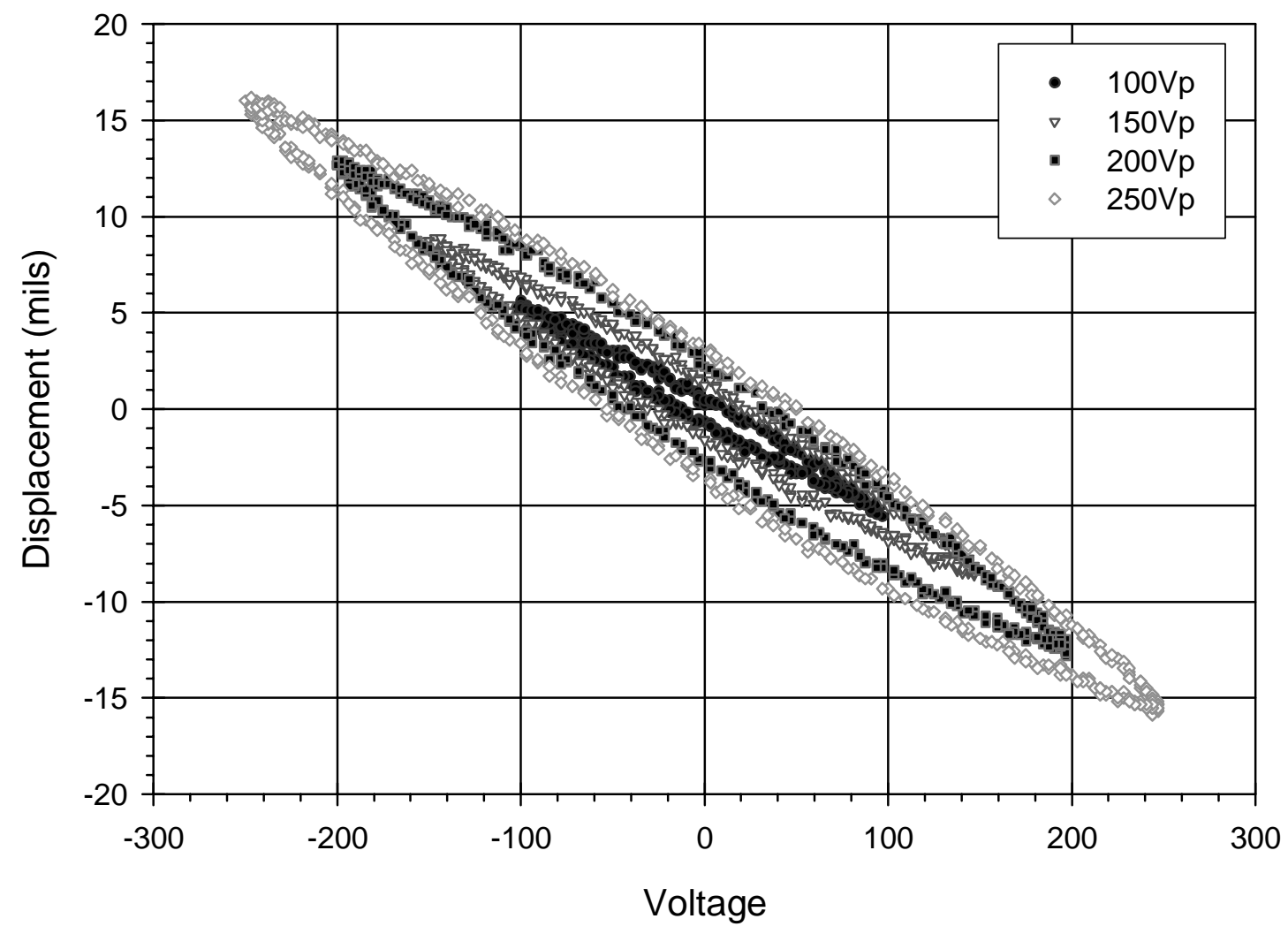

Figure 10. Typical Voltage Displacement Loop for a Lipca-C2 sample (1 Hz)

are in direct contact with the piezoelectric layer. In the case of the Thunder devices, and as pointed out earlier, the field applied has to go through the metal and the adhesive layer.

Figures 12 and 13 show the effects of frequency on the shape of the displacement loop at low to moderate fields. Again, at equal voltages, the field applied to the Lipca pieces is higher than for the Thunder pieces, rendering the samples prone to depoling. The displacement loop of Lipca at $130 \mathrm{~Hz}$ shows a beginning of asymmetric dipolar switching, similar to that observed in comparable pre-stressed actuators [3]. 


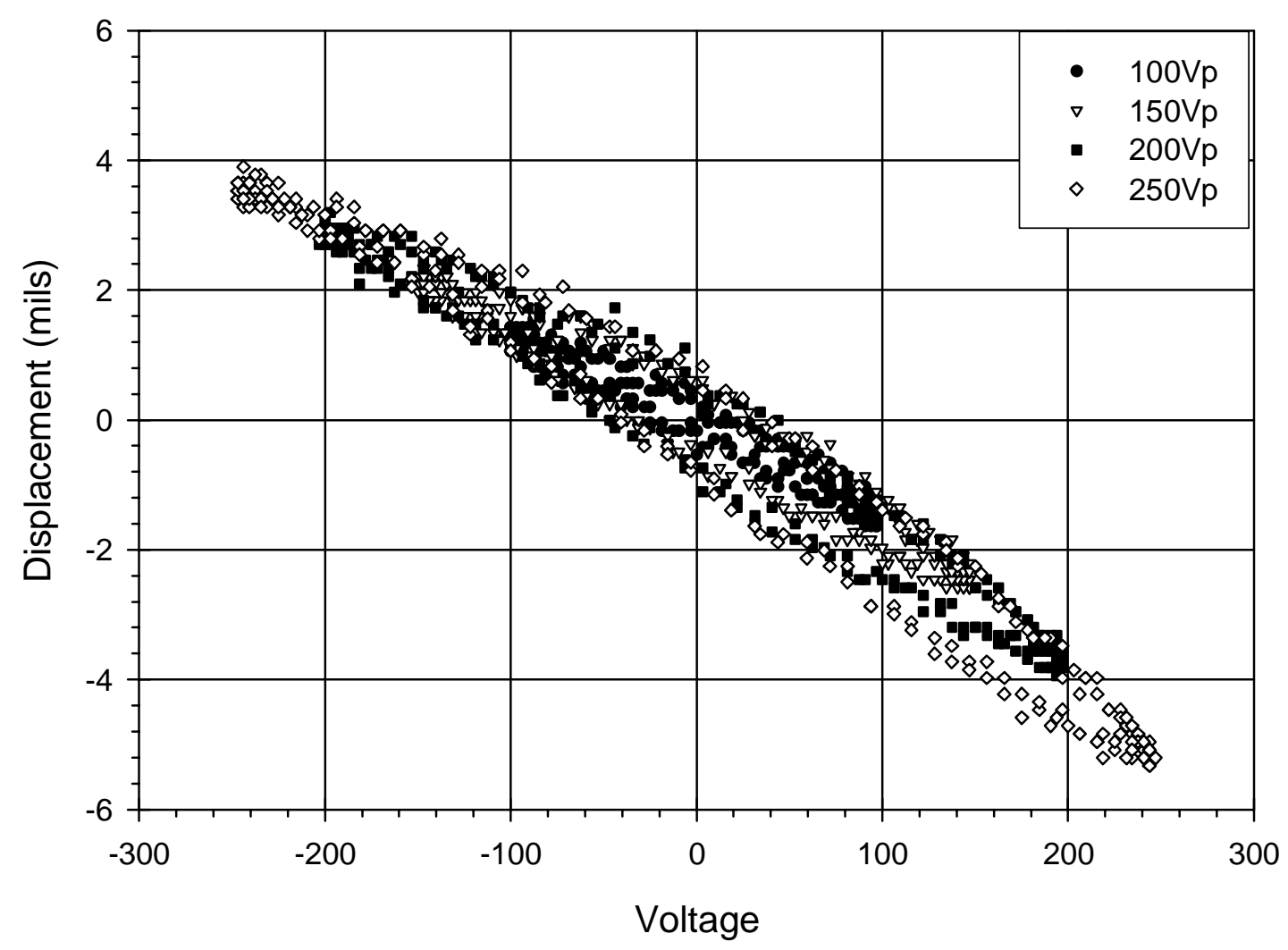

Figure 11. Typical Voltage Displacement Loop for a Lipca-C sample

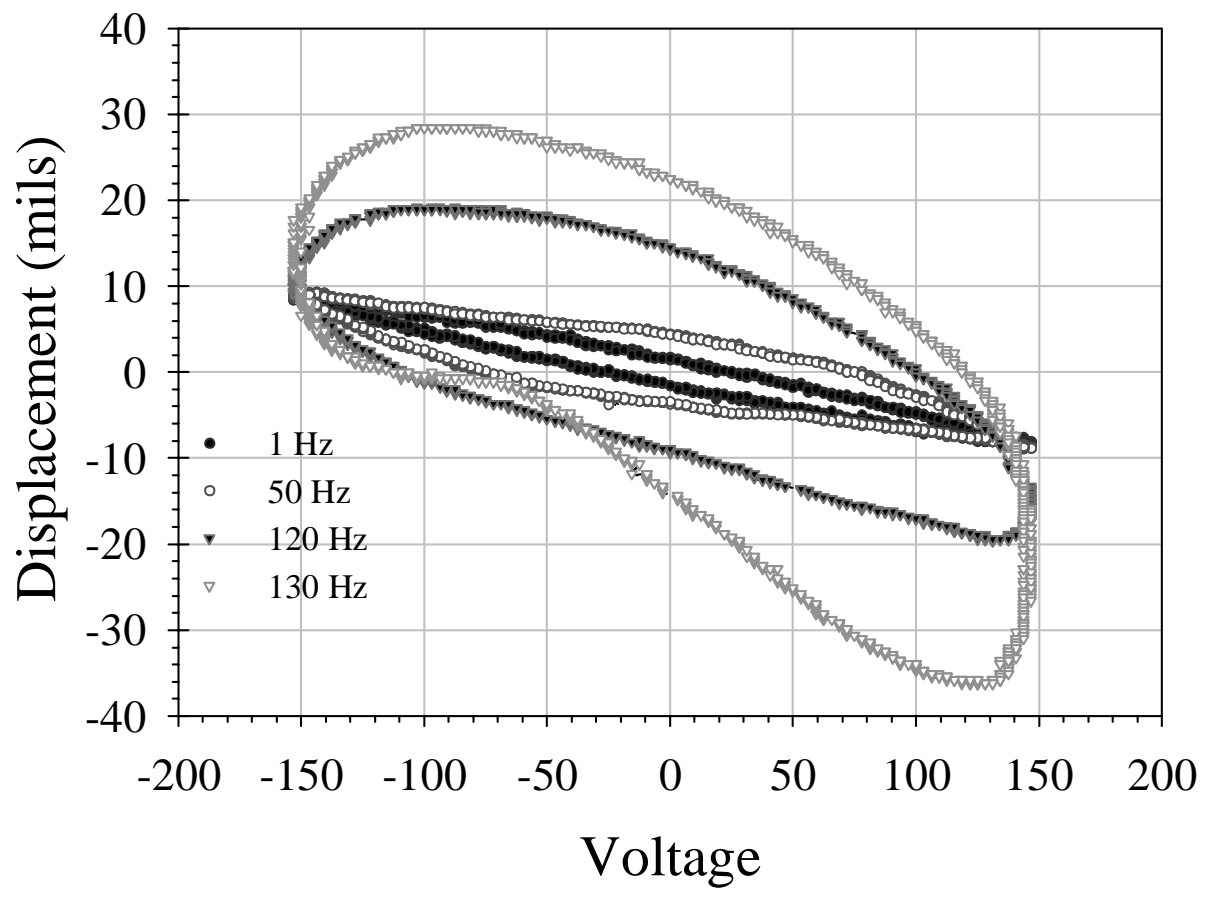

Figure 12. Typical frequency response for a Lipca sample at selected frequencies 


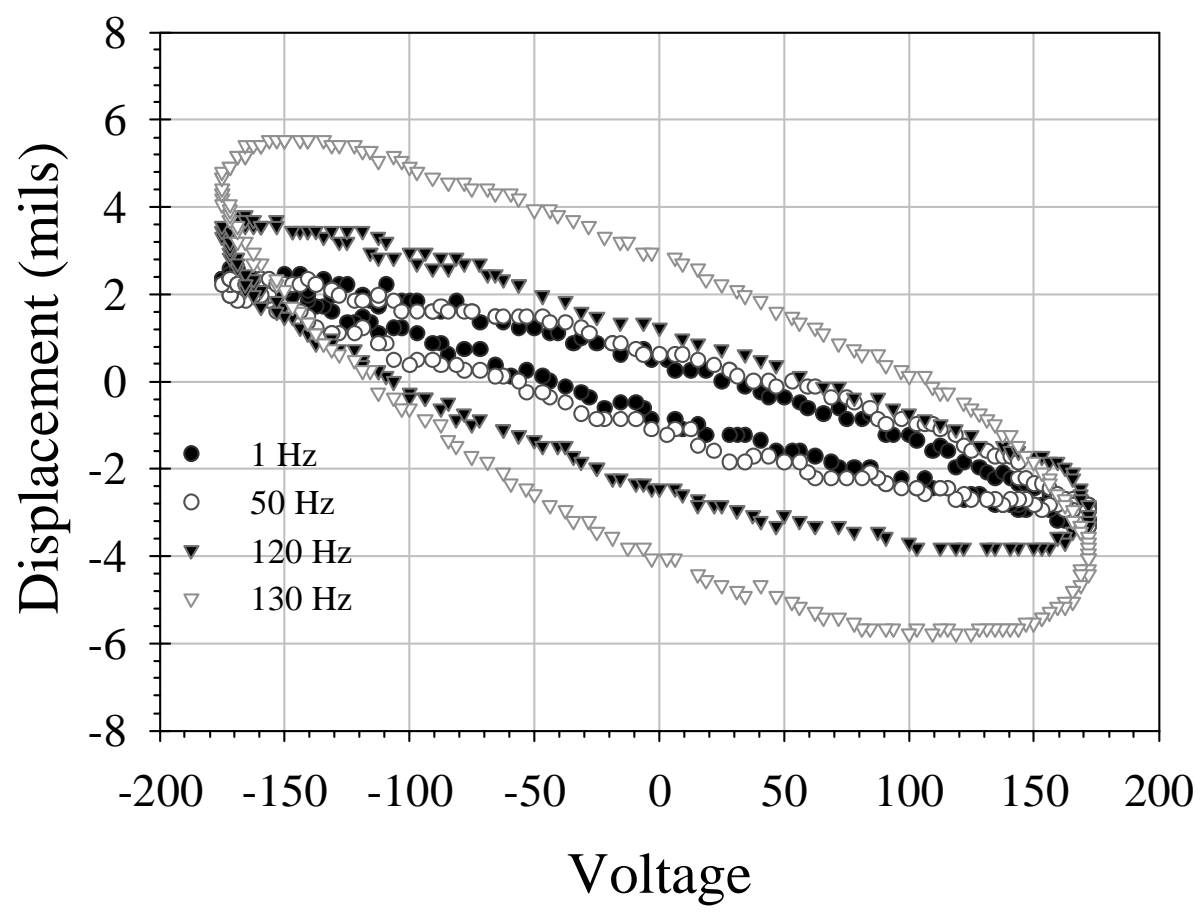

Figure 13. Typical frequency response for a Thunder sample at selected frequencies

In order to observe the frequency behavior at different voltages, maximum positive and negative displacements are read from the measured voltage displacement loops. These results are shown in Figures 14 and 15 for a Lipca and a Thunder piece respectively. It is important to note that resonant frequency shifts with applied voltage. More specifically, as voltage increased, the resonant frequency decreased indicating that the piezoelectric material softens with increasing voltage [3]. This effect can be modeled using a regression function as shown below,

$$
\delta=\delta_{0}+\frac{a}{\left[1+\left(\frac{f-f r}{b}\right)^{2}\right]}
$$

where $\delta_{\mathrm{o}}$ is the maximum displacement, constant, $a$ is a constant that depends on the voltage applied; $f$ is the frequency, $f_{r}$ is the resonant frequency; and $b$ a constant. The regression curves showed an adjusted regression factor of more than $95 \%$ for all of the Lipca and Thunder devices. A sample of the parameters obtained is shown in Table 2 for a Thunder device. Note that the resonant frequency varies linearly with the voltage applied (Figure 16 and 17). Using the linear relationship obtained, resonant frequency for both devices can be predicted at any voltage level applied. To confirm the validity of these results, using a voltage of $1 \mathrm{Vrms}(2.828 \mathrm{Vpp})$, the resonant peak of Lipca is calculated to be at $162 \mathrm{~Hz}$ and that of Thunder is at $135 \mathrm{~Hz}$. These values are in good agreement with the resonance measured using the impedance curves.

Table 2. Thunder regression parameters

$\begin{array}{lcccccc}\mathbf{V}_{\mathbf{P}} & \mathbf{1 0 0} & \mathbf{1 2 5} & \mathbf{1 5 0} & \mathbf{1 7 5} & \mathbf{2 0 0} & \mathbf{2 5 0} \\ \mathbf{R} & 0.99 & 0.996 & 0.98 & 0.98 & 0.99 & 0.98 \\ \mathbf{a} & -23.45 & -26.05 & -35.5 & -35.98 & -37.33 & -47.99 \\ \mathbf{b} & 5.51 & 6.97 & 5.87 & 6.44 & 7.40 & 8.10 \\ \mathbf{f}_{\mathbf{r}} & 130.7 & 129.4 & 127.2 & 126.9 & 126.5 & 122.9 \\ \boldsymbol{\delta}_{\mathbf{o}} & -2.3 & -2.49 & -3.2 & -3.89 & -4.137 & -5.09\end{array}$




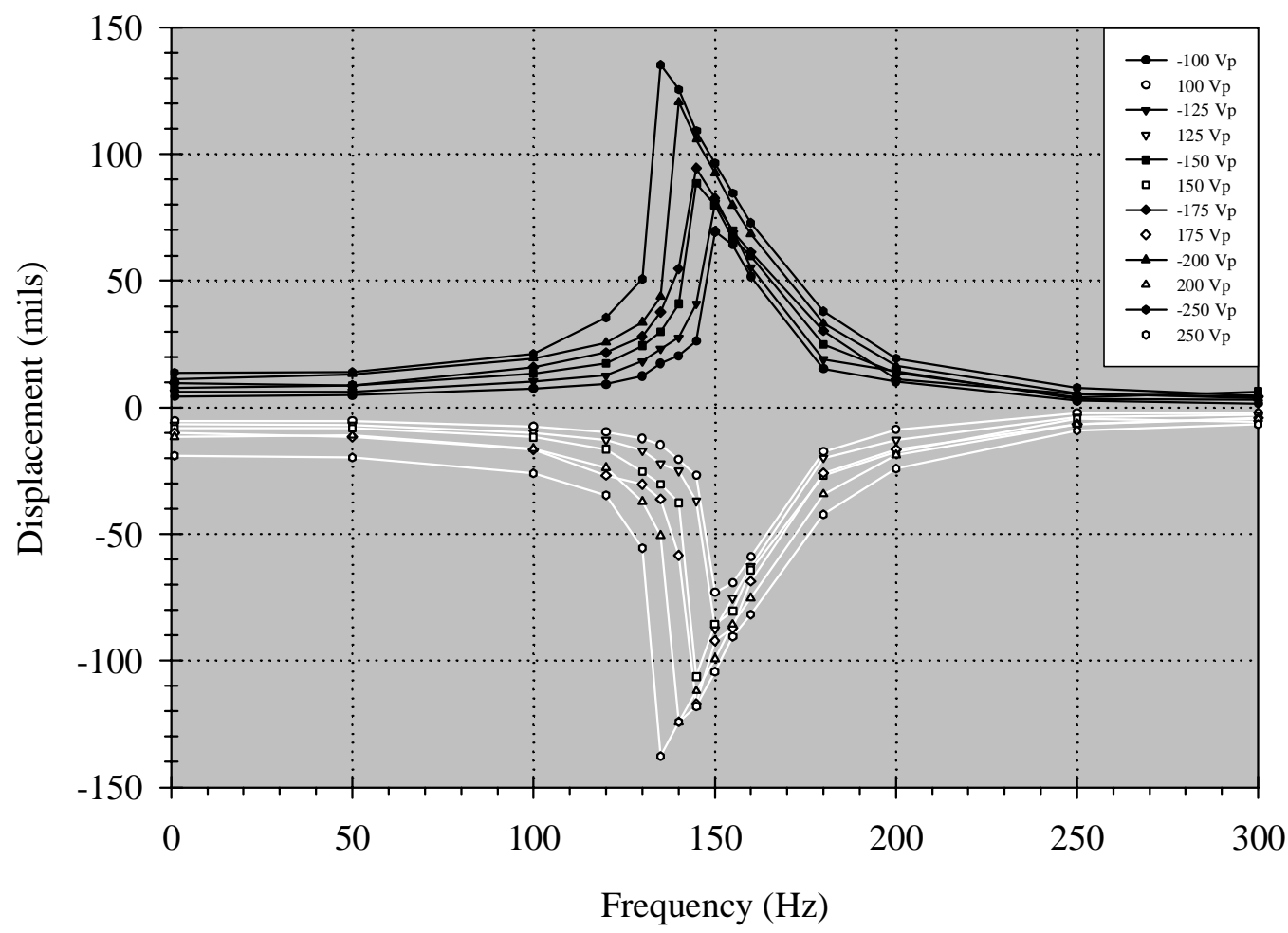

Figure 14. Peak displacement variations with frequency for a typical Lipca sample

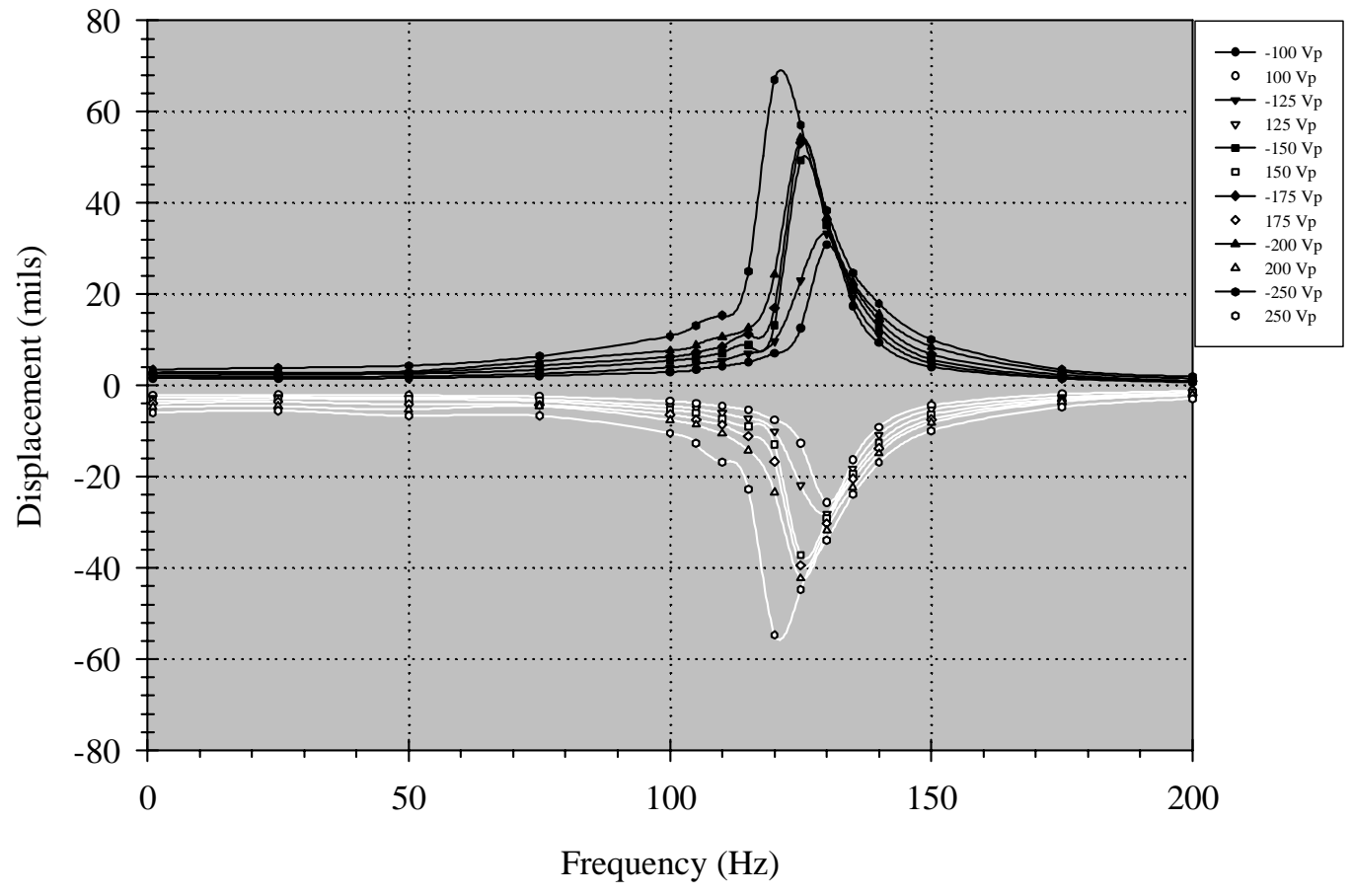

Figure 15. Peak displacement variations with frequency for a typical Thunder sample 


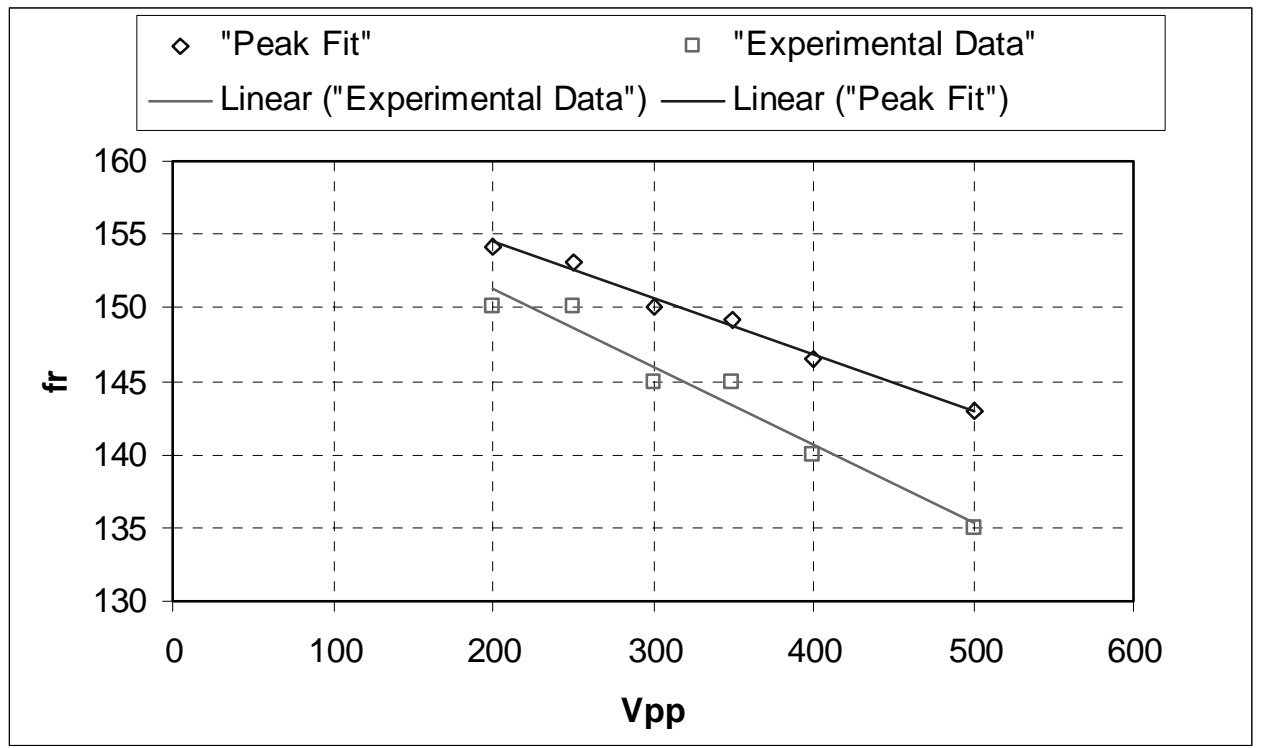

Figure 16. Resonant frequency vs. voltage for a Lipca device

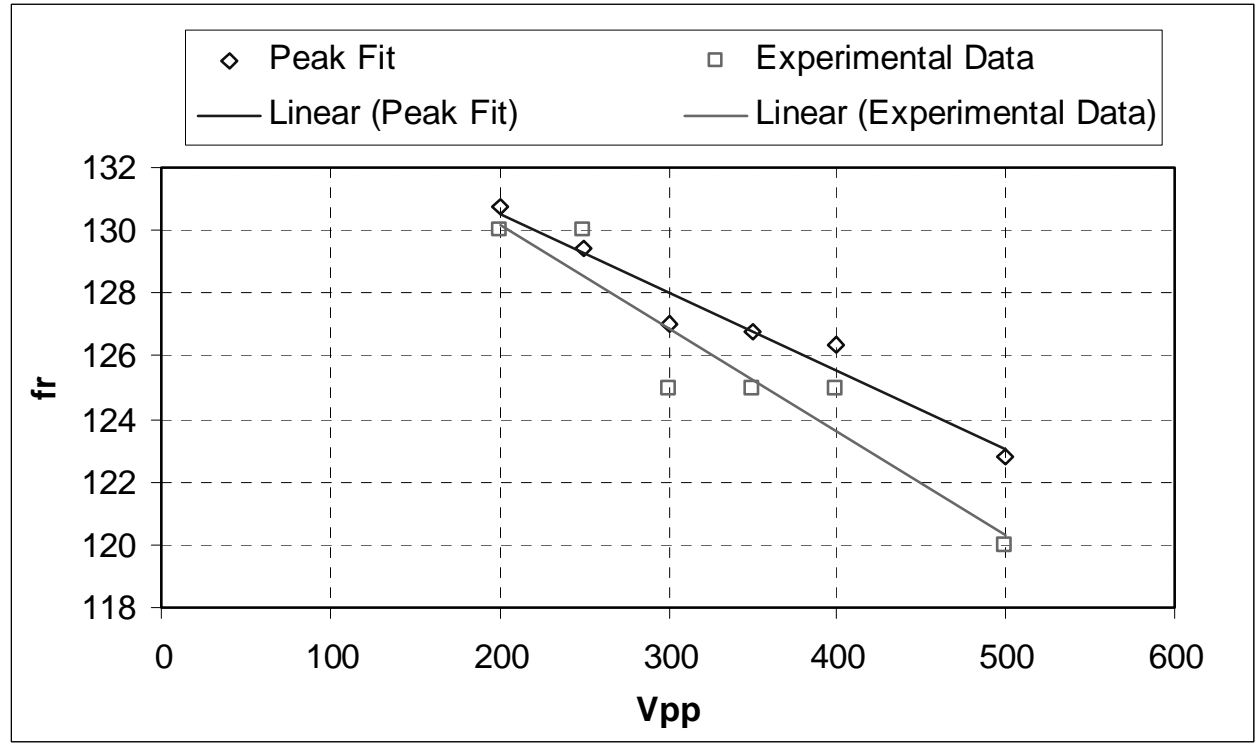

Figure 17. Resonant frequency vs. voltage for a Thunder device

A nonlinear model, which quantifies the displacements generated in THUNDER actuators in response to applied voltages for a variety of boundary conditions and exogenous loads, is developed. This model uses a PDE model based on Newtonian principles to quantify the displacements in the actuator due to field inputs to the Thunder patch stress-strain. The geometry of the device is described in Figure 18 assuming the Thunder device has metal extensions. 


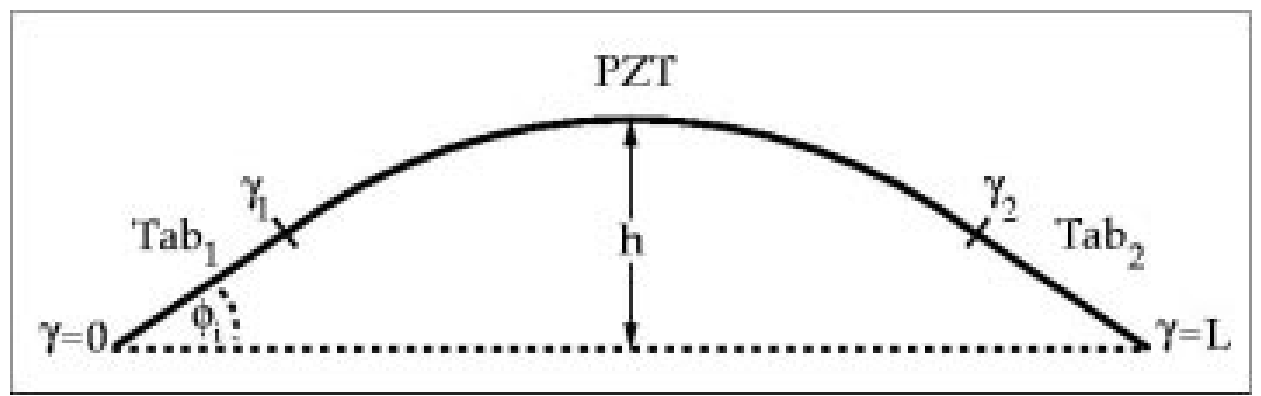

Figure 18. Boundary Conditions

A free energy based hysteretic stress-strain relation is employed to model the hysteresis inherent to the PZT polarization-electric field. The validity of the model was tested and results are shown in Figure 19.

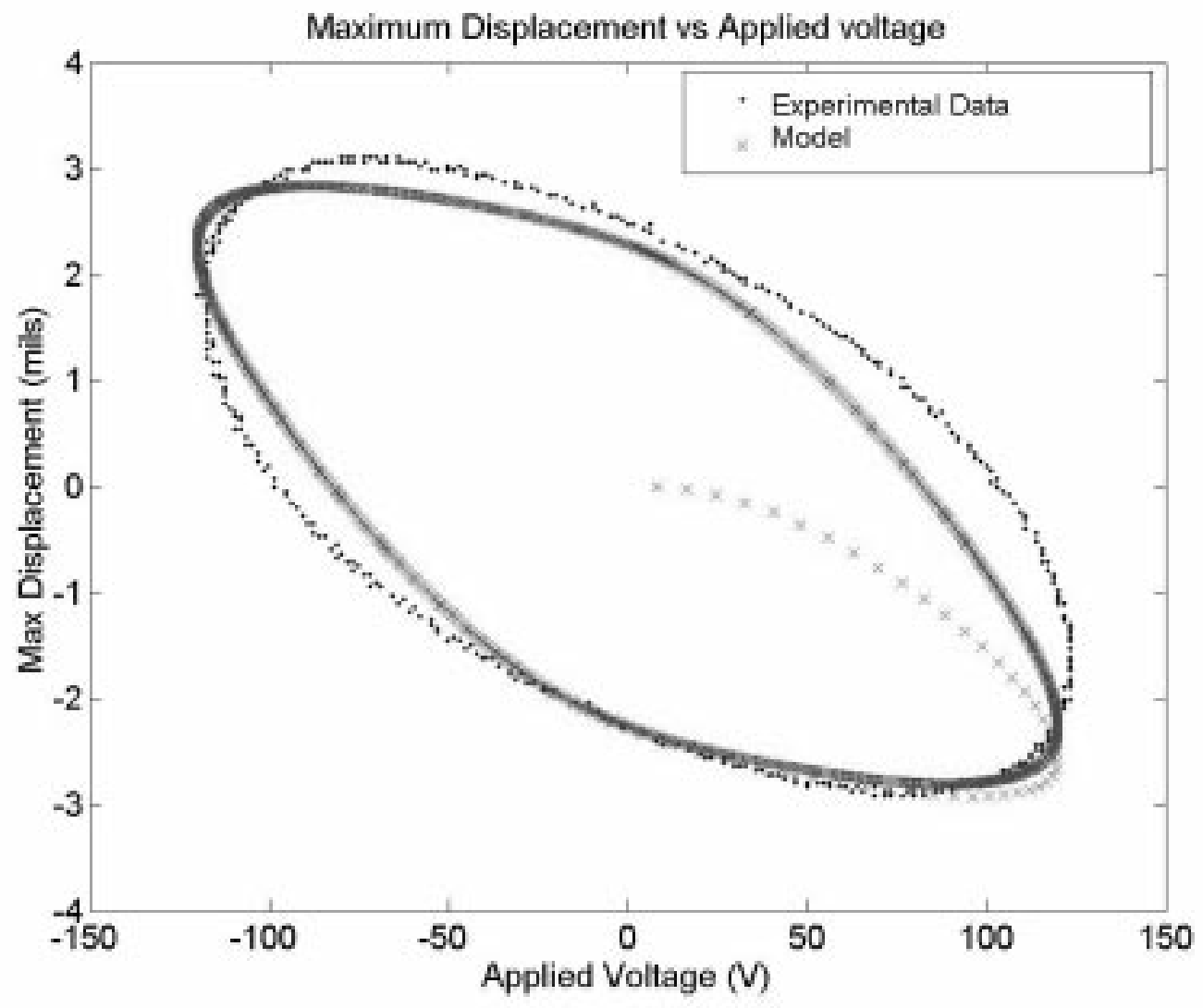

Figure 19. Maximum displacement with applied voltage 


\section{CONCLUSIONS}

Controlled experiments were performed on pre-stressed piezoelectric actuators of approximately the same physical dimensions but with different layer configurations. Lipca-C2 devices are made with fiberglass and carbon, and Thunder devices are made with stainless steel and aluminum. The performance of the devices was assessed by measuring the physical characteristics such as thickness, dome height, and weight; the dielectric properties, such as dielectric loss factor at a range of frequencies; and the out-of-plane displacement, evaluated using a simply supported pin-roller configuration at various voltages (100Vp to $250 \mathrm{Vp}$ ) and frequencies (1-300 Hz). The results showed that the Lipca devices are not just lighter, but their displacement performance is higher than the Thunder devices. Although dome height is a good predictor of the level of free displacement in Thunder, this same finding did not translate when comparing Lipca to Thunder.

Thunder samples demonstrated a more consistent performance suggesting that the manufacturing methods are more controlled than for the Lipca devices. Since stiffness was not measured, loaded displacement performance cannot be assessed. The free displacement for the Lipca devices is approximately 3 times higher than that of Thunder devices, suggesting that to a limit, Lipca devices outperform Thunder devices. For heavier loads, stiffness may play a large role. Future studies will contrast both devices under load.

\section{ACKNOWLEDGEMENTS}

The authors gratefully acknowledge helpful discussions with Professors Park and Yoon of Konkuk University. Lipca-C2 actuators used in this study were kindly provided by Konkuk University.

\section{REFERENCES}

i. Mossi, K. M., and R. P. Bishop. 1999. Characterization of Different types of High Performance THUNDER Actuators. Proc. of SPIE Conference, 1-5 March/ Newport Beach, CA, Vol 3675-05.

ii. Lobontiu, N., Goldfarb, M., Garcia, E. “A piezoelectric-driven inchworm locomotion device," Mechanism and Machine Theory 36 (2001) 425-443

iii. Ounaies, Z., Mossi, K., Smith, R., Berndt, J. “ Low-Field and High Field Characterization of Thunder Actuators," SPIE 2001 Conference, 4333-66, March 2001

iv. Wise, S.A. “ Displacement properties of RAINBOW and THUNDER piezoelectric actuators," Sensors and Actuators A, Vol. 69, 1998

v. Mossi, K., Selby, G., and Bryant, R. “ Thin-layer composite unimorph ferroelectric driver and sensor properties," Materials Letters, Vol. 35, 1998.

vi. Giurgiutiu, Victor, Gregory A. Nall, and Craig A. Rogers. 1999. Testing and Modeling of THUNDER Sensors and Actuators. SPIE's $6^{\text {th }}$ Annual International Symposium on Smart Structures and Materials, March 1-5, 1999/ Newport Beach, CA.

vii. Schwartz, R., Narayanan, M. "Development of high performance stress-biased actuators through the incorporation of mechanical pre-loads," Sensors and Actuators A 101 (2002) 322-331.

viii. Wieman, R., Smith, R., Ounaies, Z., and Bernd, J. "A displacement model for thunder actuators having general loads and boundary conditions", SPIE Proceedings: Modeling, Signal Processing and Control in Smart Structures, Vol. 4326, 2001.

ix. Yoon, K. Joon; Jae Han Chung; Nam Seo Goo; and Hoon. C. Park. 2001. Thermal Deformation and Residual Stress Analysis of Lightweight Piezo-composite Curved Actuator Device. SPIE 2001 Conference, 4333-68, March 2001.

x. Park, K.H, Kim, Y.B., Kim, Y.S., Park, H.C., Yoon, K.J. 2002. Experimental performance evaluation of lightweight piezo-composite curved actuators. SPIE 2001 Conference, 4699-41. 